\title{
Supporting information to: \\ Iron oxides control sorption and mobilisation of iodine in a tropical rainforest catchment
}

5

Laura Balzer $^{1 *}$, Katrin Schulz ${ }^{2}$, Christian Birkel ${ }^{3}$, Harald Biester ${ }^{1}$

${ }^{1}$ Department of Environmental Geochemistry, Technical University, Braunschweig, 38106, Germany

${ }^{2}$ Soil Chemistry Group, Institute of Biogeochemistry and Pollutant Dynamics, CHN, ETH, Zürich, 8092, Switzerland

${ }^{3}$ Department of Geography and Water and Global Change Observatory, University of Costa Rica, San José, Costa Rica

10 Correspondence to: Laura Balzer (laura.balzer@tu-braunschweig.de)

\section{Classification of soils in the Alberto Manuel Brenes Biological Reserve}

We classified all sampled soils in the study area as Cambisols, detailed characteristics are compiled in Table S1 for soil profiles on the left hand side of the catchment (L1-L4) and in Table S2 for soil profiles on the right hand side of the catchment. At all sites the bedrock was not found by testing to $1.5 \mathrm{~m}$. Coarser skeleton $(>2 \mathrm{~mm})$ was rare $(<10 \%)$ and the

15 top $10 \mathrm{~cm}$ of all profiles were highly penetrated by fine roots. The soil texture was determined by Roland Prietz (Thünen Institute of Climate-Smart Agriculture, Braunschweig, Germany) using finger texturing. Finger texturing was shown to be an appropriate alternative to laboratory texture analysis methods (Vos et al., 2016). Carbon and nitrogen contents in solid samples were measured using an elemental analyzer (EuroEA 3000). 
Table S1: Soil characteristics for profiles L1-L4 according to the WRB classification system.

\begin{tabular}{|c|c|c|c|c|c|c|c|c|}
\hline $\begin{array}{l}\text { Profile } \\
\text { Soil type }^{l}\end{array}$ & Horizon $^{2}$ & $\begin{array}{l}\text { Depth } \\
{[\mathrm{cm}]}\end{array}$ & Texture & $\begin{array}{l}C \\
{\left[g \mathrm{~kg}^{-1}\right]}\end{array}$ & $\begin{array}{l}C / N \\
{[-]}\end{array}$ & $\begin{array}{l}p \mathrm{H} \\
\left(\mathrm{CaCl}_{2}\right)\end{array}$ & $\begin{array}{l}\text { Grav. water } \\
\text { content [\%] }\end{array}$ & $\begin{array}{l}\text { Munsell } \\
\text { colour }\end{array}$ \\
\hline L1 & $\mathrm{Ah}$ & & & 112 & 10 & & & $10 \mathrm{YR}-2 / 2$ \\
\hline \multicolumn{9}{|l|}{ Colluvic } \\
\hline \multirow[t]{2}{*}{ Cambisol } & & $0-10$ & Silt loam & & & - & - & \\
\hline & AhBw & $>10$ & Silt loam & 58 & 16 & - & - & $10 Y R-3 / 4$ \\
\hline L2 & $\mathrm{Ah}$ & $0-12$ & & 338 & 17 & & & $10 \mathrm{YR}-2 / 2$ \\
\hline \multicolumn{9}{|l|}{ Haplic } \\
\hline \multirow[t]{4}{*}{ Cambisol } & & & Silt loam & & & 4.1 & 18 & \\
\hline & $\mathrm{AhBw}$ & $12-30$ & Silt loam & 119 & 12 & 4.6 & 14 & $10 \mathrm{YR}-3 / 2$ \\
\hline & $\mathrm{Bw}$ & $30-70$ & Silt loam & 30 & 7 & 4.9 & 13 & $10 \mathrm{YR}-3 / 4$ \\
\hline & $2 \mathrm{Bw}$ & $>70$ & Silt loam & 16 & 7 & 4.4 & 11 & $10 \mathrm{YR}-3 / 4$ \\
\hline L3 & $\mathrm{Ah}$ & $0-7$ & Silt loam & 192 & 14 & & & 10YR-2/1 \\
\hline \multicolumn{9}{|l|}{ Colluvic } \\
\hline \multirow[t]{4}{*}{ Cambisol } & & & & & & - & - & \\
\hline & $\mathrm{AhBw}$ & $7-26$ & Silt loam & 117 & 12 & - & - & $10 \mathrm{YR}-2 / 2$ \\
\hline & $2 \mathrm{Bw}$ & $>26$ & Silty clay & 23 & 6 & & & $10 \mathrm{YR}-4 / 3$ \\
\hline & & & loam & & & - & - & \\
\hline \multirow{5}{*}{$\begin{array}{l}\text { L4 } \\
\text { Colluvic } \\
\text { Cambisol }\end{array}$} & $\mathrm{Ah}$ & $0-10$ & Silt loam & 187 & 12 & & & $10 \mathrm{YR}-2 / 1$ \\
\hline & & & & & & & & \\
\hline & & & & & & - & - & \\
\hline & $\mathrm{AhBw}$ & $10-35$ & Silt loam & 60 & 9 & - & - & $10 \mathrm{YR}-2 / 2$ \\
\hline & $2 \mathrm{Bw}$ & $>35$ & Silt loam & 42 & 7 & - & - & 10YR-3/4 \\
\hline
\end{tabular}

1 Soil types according to WRB classification: Food and Agriculture Organization (FAO, 2014),

2 Soil horizons according to FAO (2006): Guidelines for Soil Description 
Table S2: Soil characteristics for profiles R1-R5 according to the WRB classification system.

\begin{tabular}{|c|c|c|c|c|c|c|c|c|}
\hline $\begin{array}{l}\text { Profile } \\
\text { Soil type }\end{array}$ & Horizon $^{2}$ & $\begin{array}{l}\text { Depth } \\
{[\mathrm{cm}]}\end{array}$ & Texture & $\begin{array}{l}C \\
{\left[g_{k g}^{-1}\right]}\end{array}$ & $\begin{array}{l}\text { C/N } \\
{[-]}\end{array}$ & $\begin{array}{l}p \mathrm{H} \\
\left(\mathrm{CaCl}_{2}\right)\end{array}$ & $\begin{array}{l}\text { Grav. water } \\
\text { content [\%] }\end{array}$ & $\begin{array}{l}\text { Munsell } \\
\text { colour }\end{array}$ \\
\hline \multicolumn{9}{|l|}{ R1 } \\
\hline \multirow[t]{2}{*}{ Cambisol } & $\mathrm{Ah}$ & $0-8$ & Sandy loam & 129 & 12 & - & - & - \\
\hline & $\mathrm{Bw}$ & $>8$ & Sandy loam & 33 & 7 & - & - & - \\
\hline \multicolumn{9}{|l|}{$\mathbf{R 2}$} \\
\hline Haplic & & & Silt loam & & & 4.7 & 14 & \\
\hline \multirow[t]{3}{*}{ Cambisol } & $\mathrm{Ah}$ & $0-10$ & & 100 & 11 & & & 10YR-2/1 \\
\hline & $\mathrm{AhBw}$ & $10-30$ & Silt loam & 64 & 9 & 4.8 & 14 & $10 \mathrm{YR}-2 / 2$ \\
\hline & $\mathrm{Bw}$ & $>30$ & Silt loam & 25 & 6 & 4.4 & 13 & $10 Y R-3 / 4$ \\
\hline \multicolumn{9}{|l|}{$\mathbf{R 3}$} \\
\hline \multirow[t]{4}{*}{ Cambisol } & $\mathrm{Ah}$ & $0-13$ & Silt loam & 112 & 12 & - & - & $10 \mathrm{YR}-2 / 2$ \\
\hline & $\mathrm{Bw}$ & $13-32$ & Silt loam & 47 & 8 & - & - & 10YR-3/4 \\
\hline & $2 \mathrm{Bw}$ & $>32$ & Silty clay & & & & & $10 Y R-3 / 4$ \\
\hline & & & loam & 27 & 6 & - & - & \\
\hline \multicolumn{9}{|l|}{$\mathbf{R 4}$} \\
\hline Dystric & & & & & & 4.3 & - & \\
\hline \multirow[t]{2}{*}{ Cambisol } & $\mathrm{Ah}$ & $0-15$ & Silt loam & 132 & 12 & & & $10 \mathrm{YR}-2 / 2$ \\
\hline & $\mathrm{Bw}$ & $>15$ & Silt loam & 29 & 7 & 4.4 & - & $10 \mathrm{YR}-3 / 4$ \\
\hline \multicolumn{9}{|l|}{ R5 } \\
\hline \multirow[t]{2}{*}{ Cambisol } & $\mathrm{Ah}$ & $0-8$ & Silt loam & 109 & 11 & - & - & $10 \mathrm{YR}-2 / 2$ \\
\hline & $\mathrm{Bw}$ & $>8$ & Silt loam & 42 & 7 & - & - & $10 \mathrm{YR}-3 / 4$ \\
\hline
\end{tabular}

1 Soil types according to WRB classification: Food and Agriculture Organization (FAO, 2014),

2 Soil horizons according to FAO (2006): Guidelines for Soil Description

\section{References}

Vos, C., Don, A., Prietz, R., Heidkamp, A., and Freibauer, A.: Field-based soil-texture estimates could replace laboratory analysis, Geoderma, 267, 215-219, https://doi.org/10.1016/j.geoderma.2015.12.022, 2016. 